\title{
Die Arbeitszeitfrage im Kontext von Wachstum, Ökologie und Wohlstand
}

\author{
Wirtschaftliches Wachstum und wachsender \\ Wohlstand galten lange Zeit als zwei Seiten einer \\ Medaille. Doch geringes Wachstum verschärft die \\ Situation am Arbeitsmarkt. Arbeitszeitverkürzung \\ erscheint als Königsweg, den Wohlstand der \\ Menschen weiter zu steigern, ohne die Umwelt \\ weiter zu belasten. \\ Von Norbert Reuter
}

$\mathrm{H}$ eute ist es fast schon ein Allgemeinplatz, dass das Bruttoinlandsprodukt bereits als Messgröße für die Leistung einer Volkswirtschaft erhebliche Defizite aufweist (Deutscher Bundestag 2013). Wegen der zunehmend negativen Begleiterscheinungen von lang anhaltenden Wachstumsphasen, nämlich sich zuspitzenden Umweltproblemen, Grenzen natürlicher Ressourcen, wachsender Überfluss, zunehmender Stress und Arbeitsdruck, ist das Bruttoinlandsprodukt (BIP) als Wohlstandsmaß gerade in fortgeschrittenen Industrieländern sogar weitgehend unbrauchbar geworden (Deutscher Bundestag 2013).

Diese Kritik an der üblichen Wohlstandsmessung war auch eine der Ursachen für die Einsetzung der Enquete-Kommission „Wachstum, Wohlstand, Lebensqualität“ durch den Deutschen Bundestag von 2011 bis 2013.

\section{Lebensqualität durch Fortschritt?}

Das grundsätzliche Problem ist, dass sich aus dem anhaltenden Produktivitätsfortschritt die Notwendigkeit ergibt zu entscheiden, wie mit der hieraus resultierenden höheren Wertschöpfung umgegangen werden soll. Wird die höhere Wertschöpfung für eine steigende Produktion genutzt, ist Wachstum mit allen skizzierten Problemen die Folge. Alternativ bleibt nur eine sukzessive Arbeitszeitverkürzung. Eine Verkürzung der Arbeitszeit findet sich als Empfehlung im Abschlussbericht der Enquete-Kommission, da dies die "sozialste und ökologischste Form der Wohlstandssteigerung sei“ (Deutscher Bundestag 2013).

Ergänzend wird darauf hingewiesen, dass die jährliche Reduktion der Arbeitszeit um 0,5\% bis zum Jahr 2100 dazu beitragen könnte, „die globale Erwärmung zwischen einem Viertel und der Hälfte“ zu reduzieren (Deutscher Bundestag 2013).

\section{Das Comeback der Arbeitszeitfrage}

Die Anerkennung vorhandener Grenzen des Wachstums bei gleichzeitig abnehmendem Nutzen beziehungsweise abnehmendem Sinn einer sich weiter steigernden Produktion von Gütern und zunehmenden Umweltproblemen erfordert eine Fokussierung auf qualitative Entwicklung statt quantitatives Wachstum. Sobald nicht mehr ein eindimensionales BIPWachstum, sondern eine mehrdimensionale Wohlstandsentwicklung das Ziel ist, ergibt sich die Notwendigkeit, die Bedingungen und Inhalte von Wohlstand in einem demokratischen Prozess zu definieren. Dieser mündet in der Frage, welche Gesellschaft wir wollen.

Auch aus gewerkschaftlicher Sicht spielt in diesem Zusammenhang die Frage der Arbeitszeiten wieder eine größere Rolle. Hierfür sind vor allem zwei Gründe verantwortlich. Erstens die nach wie vor bestehende Massenarbeitslosigkeit und zweitens, die gleichzeitig vorhandenen Wünsche der Beschäftigten nach reduzierten Arbeitszeiten.

\section{Arbeitszeitverkürzung zum Abbau der Massenarbeitslosigkeit}

Trotz aller Erfolgsmeldungen vom Arbeitsmarkt und statistischen Beschönigungen sind auch im Herbst 2015 tatsächlich immer noch gut 3,6 Millionen Menschen in Deutschland arbeitslos. Trotz enormer Exportüberschüsse hat sich mit Blick auf die insgesamt geleisteten Arbeitsstunden der Erwerbstätigen kaum etwas getan. Auch wenn seit 2005 ein tendenzieller Anstieg des Arbeitsvolumens zu verzeichnen ist, bewegt es sich mit rund 58 Milliarden Arbeitsstunden heute immer noch auf dem Niveau von Anfang der 1990er Jahre.

Aufgrund der anhaltenden Produktivitätssteigerung wäre heute aber ein Wachstum des BIP von derzeit rund 1,4\%, Durchschnitt der letzten zwei Dekaden, pro Jahr notwendig, damit lediglich das vorhandene Arbeitsvolumen erhalten bleibt und keine weiteren Arbeitsplätze verloren gehen. Da aber die Wachstumsraten in allen entwickelten Ländern sich auf niedrigem Niveau bewegen, in der Tendenz sogar weiter sinken (Reuter 2000) und die ökologischen Probleme schon jetzt kaum beherrschbar sind, erscheint die Überwindung der Massenarbeitslosigkeit über den Wachstumspfad utopisch. Eine Rückkehr auf den Pfad einer gesamtwirtschaftlichen Arbeitszeitverkürzung, der Ende der 1990er Jahre ein Ende hatte, erscheint vor diesem Hintergrund als alternativlos (siehe hierzu auch Arbeitsgruppe Alternative Wirtschaftspolitik 2012). 


\section{Arbeitszeitverkürzung für mehr Zeitwohlstand}

Neben Arbeitszeitverkürzung zum Abbau der Massenarbeitslosigkeit wird die Arbeitszeitfrage auch zunehmend vor dem Hintergrund von Wohlstandsüberlegungen diskutiert. Grundsätzlich eröffnen sich auf hohem Einkommensniveau in besonderer Weise Spielräume den individuellen Wohlstand statt durch Einkommenssteigerungen durch Arbeitszeitverkürzung zu steigern. Der Nutzen zusätzlicher Freizeit steigt hier tendenziell über den Nutzen zusätzlicher Einkommen.

Hierauf weisen zum einen immer wieder Berichte von Beschäftigten hin, die im Zuge von krisenbedingter Kurzarbeit eine Zeit lang kürzer arbeiten mussten. Nach den Erfahrungen einer länger anhaltenden Vier-Tage-Woche fällt es offensichtlich schwer, diesen Zugewinn an Freizeit und Lebensqualität wieder aufzugeben. Dies trifft auch zu, obwohl die Einkommen nach Ende der Kurzarbeit wieder auf das alte Niveau steigen. Diese anekdotische Evidenz wird durch Arbeitszeitexperimente bestätigt. Unternehmensberater in den USA, die gezwungen wurden, einen Tag in der Woche freizunehmen, bewerteten nach fünf Monaten ihre Arbeits- und Lebenssituation deutlich besser als ihre Kollegen ohne Arbeitszeitverkürzung (Kopatz 2012).

\section{Politische Folgerungen}

Vor dem Hintergrund zunehmender Umweltprobleme als Folge wirtschaftlichen Wachstums kommt politischen Strategien eine herausragende Bedeutung $\mathrm{zu}$, die geeignet sind, den Wohlstand der Menschen weiter zu steigern, ohne die Umwelt weiter zu belasten. Arbeitszeitverkürzung erscheint hier geradezu als Königsweg. Allerdings ist ein einhelliger Wunsch der Beschäftigten nach Arbeitszeitverkürzung nicht erkennbar. Während Vollzeitbeschäftigte weniger arbeiten wollen, äußern vor allem weibliche Teilzeitbeschäftigte den Wunsch, länger zu arbeiten. Gewünscht wird im Durchschnitt eine wöchentliche Arbeitszeit von rund 35 Stunden.

Die Forderung nach Arbeitszeitverkürzung würde vermutlich noch deutlicher artikuliert, hätten Beschäftigte nicht vielfach negative Erfahrungen mit der Verkürzung der Arbeitszeit gemacht. Für sie hat sich das Arbeitspensum nämlich nicht reduziert, sodass die Arbeitsbelastung gestiegen ist. Durch die Veränderung der Rahmenbedingungen könnte Arbeitszeitverkürzung attraktiver gemacht werden:

- Beschäftigte müssen die rechtliche Garantie haben, nach gewähltem Übergang in Teilzeit gegebenenfalls auch wieder zurück in Vollzeit wechseln zu können.

- Im Rahmen von Tarifverhandlungen sind statt kollektiver Lohn- und Arbeitszeitregelungen individuelle Wahlmöglichkeiten zwischen Arbeitszeitverkürzung und Lohnerhöhung zu schaffen. Dergleichen gibt es bereits beispielsweise in Tarifverträgen der Elektro- und Elektronikindustrie und der Fahrzeugindustrie in Österreich.
- Arbeitszeitverkürzung muss immer mit klaren Regelungen zur Verkürzung des Arbeitspensums beziehungsweise mit einem vollumfänglichen Personalausgleich einhergehen, damit ein steigender Leistungsdruck vermieden wird.

- Entsprechend der arbeitszeitpolitischen Wünsche der Beschäftigten müssen Arbeitszeitmodelle sich an der Perspektive „Kurze Vollzeit für alle“ (Zimpelmann/Endl 2008) orientieren. Die unterschiedlichen Wünsche von Teil- und Vollzeitbeschäftigten sind demnach zu berücksichtigen (vgl. hierzu den Beitrag von Sabine Reiner in diesem Heft).

Flankierend müsste die sich ausbreitende qualitative Verschlechterung des Produktivitätsfortschritts und damit der Arbeitsbedingungen zurückgedrängt werden. Eine zunehmende Arbeitshetze und -intensivierung könnte verhindert beziehungsweise bekämpft werden, indem entsprechende Personalschlüssel und Pausenregelungen vereinbart werden, unbezahlte Mehrarbeit verhindert, auf eine klare Trennung zwischen Arbeitszeit und Freizeit geachtet und Gesundheitsvorsorge großgeschrieben wird. Ein willkommener Nebeneffekt wäre ein niedrigerer Produktivitätsfortschritt, sodass ein geringeres Wachstum zum Erhalt von Arbeitsplätzen notwendig würde.

Auch wenn Arbeitszeitverkürzung sich als Königsweg darstellt, um wirtschaftliche Entwicklung, Ökologie und Wohlstand miteinander zu versöhnen, setzt sie sich nicht von alleine durch. Richtige Rahmensetzungen auf unterschiedlichen Ebenen, Aufklärung über die Folgen ungebremsten Wachstums und ein anhaltender Wertewandel sind notwendig, damit Zeitwohlstand eine zunehmende Bedeutung im Rahmen der wirtschaftlichen Entwicklung erlangt.

\section{Literatur \\ Arbeitsgruppe Alternative Wirtschaftspolitik (2012): Memorandum 2012. Europa am Scheideweg. Köln. \\ Deutscher Bundestag (2013): Enquete-Kommission „Wachstum, Wohlstand Lebensqualität - Wege zu nachhaltigem Wirtschaften und gesellschaft- lichem Fortschritt in der Sozialen Marktwirtschaft". Schlussbericht. Berlin. Kopatz, M. (2012): Arbeit, Glück und Nachhaltigkeit. Wuppertal. \\ Reuter, N. (2000): Ökonomik der „Langen Frist“. Zur Evolution der Wachs- tumsgrundlagen in Industriegesellschaften. Marburg. \\ Zimpelmann, B./Endl, H.-L. (2008): Zeit ist Geld. Ökonomische, ökologische und soziale Grundlagen der Arbeitszeitverkürzung. Hamburg.}

\section{AUTOR + KONTAKT}

PD Dr. Norbert Reuter ist Gewerkschaftssekretär bei ver.di, lehrt Volkswirtschaftslehre an der RWTH Aachen und war Mitglied der Enquete-Kommission „Wachstum, Wohlstand Lebensqualität“.

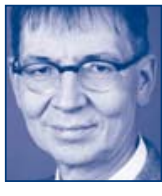

ver.di Bundesvorstand, Ressort 1, Wirtschaftspolitik, Paula-Thiede-Ufer 10, 10179 Berlin Tel.+49 30 69561136, E-Mail: norbert.reuter@verdi.de 